\title{
Article \\ Metabolic Profiling of Sugars and Organic Acids, and Expression Analyses of Metabolism-Associated Genes in Two Yellow-Peel Pitaya Species
}

\author{
Fangfang Xie ${ }^{1,2} \mathbb{D}$, Canbin Chen ${ }^{2}$, Jiaxuan Chen ${ }^{2}$, Yuanju Yuan ${ }^{2}$, Qingzhu Hua ${ }^{2}$, Zhike Zhang ${ }^{1,2} \mathbb{D}$, \\ Jietang Zhao ${ }^{1,2}$, Guibing $\mathrm{Hu}^{1,2}$, Jianye Chen ${ }^{1,2, * \mathbb{D}}$ and Yonghua Qin ${ }^{1,2, * \mathbb{D}}$ \\ 1 Guangdong Provincial Key Laboratory of Postharvest Science of Fruits and Vegetables, \\ College of Horticulture, South China Agricultural University, Guangzhou 510642, China; \\ xiefangfang202012@163.com (F.X.); poloky2@163.com (Z.Z.); zhaojietang@gmail.com (J.Z.); \\ guibing@scau.edu.cn (G.H.) \\ 2 Key Laboratory of Biology and Genetic Improvement of Horticultural Crops (South China), \\ Ministry of Agriculture and Rural Affairs, College of Horticulture, South China Agricultural University, \\ Guangzhou 510642, China; nnchencanbin@163.com (C.C.); jxchen0127@163.com (J.C.); \\ yyj974028200@163.com (Y.Y.); huaqingzhu@stu.scau.edu.cn (Q.H.) \\ * Correspondence: chenjianye@scau.edu.cn (J.C.); qinyh@scau.edu.cn (Y.Q.)
}

check for updates

Citation: Xie, F.; Chen, C.; Chen, J.; Yuan, Y.; Hua, Q.; Zhang, Z.; Zhao, J.; Hu, G.; Chen, J.; Qin, Y. Metabolic Profiling of Sugars and Organic Acids, and Expression Analyses of Metabolism-Associated Genes in Two Yellow-Peel Pitaya Species. Plants 2022, 11, 694. https://doi.org/ $10.3390 /$ plants 11050694

Academic Editor: Matteo Busconi

Received: 17 January 2022

Accepted: 1 March 2022

Published: 4 March 2022

Publisher's Note: MDPI stays neutral with regard to jurisdictional claims in published maps and institutional affiliations.

Copyright: (C) 2022 by the authors. Licensee MDPI, Basel, Switzerland. This article is an open access article distributed under the terms and conditions of the Creative Commons Attribution (CC BY) license (https:// creativecommons.org/licenses/by/ $4.0 /)$.

\begin{abstract}
Sugar and organic acids are important factors determining pitaya fruit quality. However, changes in sugars and acids, and expressions of metabolism-associated genes during fruit maturation of yellow-peel pitayas are not well-documented. In this study, metabolic and expression analyses in pulps of different fruit developmental stages of 'Wucihuanglong' ('WCHL', Hylocereus undatus) and 'Youcihuanglong' pitaya ('YCHL', Hylocereus megalanthus) were used to explore the sugar and organic acid metabolic process. Total phenols and flavonoids were mainly accumulated at $\mathrm{S} 1$ in pitaya pulps. Ascorbic acid contents of 'WCHL' pitaya were higher than that of 'YCHL' pitaya during fruit maturation. Starch was mainly accumulated at early fruit development stages while soluble sugars were rich in late stages. Sucrose, fructose, and glucose were the main sugar components of 'YCHL' pitaya while glucose was dominant in 'WCHL' pitaya. Malic and citric acids were the main organic acids in 'WCHL' and 'YCHL' pitayas, respectively. Based on the transcriptome analyses, 118 genes involved in pitaya sugar and organic acid metabolism were obtained. Results from the correlation analyses between the expression profiling of candidate genes and the contents of sugar and organic acid showed that 51 genes had a significant correlation relationship and probably perform key role in pitaya sugar and organic acid metabolism processes. The finding of the present study provides new information for quality regulation of pitayas.
\end{abstract}

Keywords: yellow-peel pitaya; sugar and organic acid metabolism; transcriptome analyses; gene expression

\section{Introduction}

Pitaya (also known as dragon fruit) belonging to Hylocereus genus within the Cactaceae family (Caryophyllales order) is native to Mexico, and Central and South America. Nowadays, pitayas are widely commercially cultivated in the tropical and subtropical regions. Based on the color of peel and pulp, pitaya is mainly classified into three species, i.e., Hylocereus undatus ( $H$. undatus, red or yellow peel with scales and white pulp), $H$. monacanthus or $H$. polyrhizus (red peel with scales and red pulp) and H. megalanthus or Selenicereus megalanthus (yellow peel without scales and with white pulp) [1]. Pitaya is popular with consumers due to its abundant betalains, exotic appearance, fresh and sweet taste, and high nutrients [2-4]. Moreover, yellow-peel pitayas have economic potential in the market due to its conspicuous appearance, shocking yellow color and long shelf life. Pitaya cultivars from a different genetic background have different biochemical and 
nutritional characteristics. In general, sugar and organic acid metabolism processes are developed during fruit development and maturation with sugar accumulation and organic acid degradation. Besides, sugar and organic acids are crucial components of pitaya fruit quality, including taste, flavor, and $\mathrm{pH}$, which influences the needs of consumers.

During fruit development and ripening, several metabolite processes are presented, of which sugar and organic metabolism perform important roles in fruit quality formation. Starch, as the predominant storage carbohydrate in plants, mainly consists of linear amylose and branched amylopectin. Starch is an important index associated with fruit texture, for instance, the softness of guava [5], and smoothness and dry constancy of pumpkin [6]. Starch synthesis and degradation pathway are involved in the regulation of fruit quality $[7,8]$. Phosphoglucomutase (PGM), ADP-glucose pyrophosphorylase (AGPS) and starch synthase (StSy) are key regulatory enzymes responsible for starch biosynthesis [8-10]. During starch metabolism, the phosphorylation of amylopectin is an essential step which requires close collaboration of dikinases, the glucan, water dikinase (GWD), and phosphoglucan, water dikinase (PWD) [7]. Simultaneously, starch is degraded while the sugars are accumulated during fruit development and ripening [3,6].

The levels of soluble sugars such as glucose, fructose, and sucrose are responsible for fruit sweetness. In citrus, sucrose, fructose, and glucose are the three major carbohydrates which is the main components of total soluble solids [11]. Glucose and fructose are the most abundant molecules in pomegranate [12] and watermelon [13]. Sucrose is the main component of soluble sugars in apricot [14] compared with pear, which is fructose-dominant [15], while red-peel pitaya is glucose-dominant $[3,16]$. Sucrose phosphate synthase (SPS) is the key enzyme responsible for sucrose synthesis while invertase (Ivr) and sucrose synthase (SuSy) are the main enzymes involved in sucrose catabolism [17,18]. Moreover, only hexokinases (HXKs) and fructokinases (FRKs) are capable of catalyzing the essential irreversible phosphorylation of glucose and fructose in plants [19]. Glucose-6-phosphate isomerase (PGI) catalyzes the interconversion between D-glucose-6-phosphate and D-fructose-6phosphate which performs important roles in glycolysis and gluconeogenesis [20].

The fruit sourness is principally determined by organic acids (mainly citric and malic acids). Citric acid and malic acid are the main organic acids in pomegranates [12]. Malic acid is dominant in red-peel pitaya [3], watermelon [13], and apple [21] while citric acid is dominant in citrus [11] and pear [22]. The glycolytic pathway is the oxidization of glucose to pyruvate, of which phosphoglycerate kinase (PGK), enolase (Eno), and pyruvate kinase (PK) are key enzymes for catalyzing 1,3-bisphosphoglycerate (1,3-BPGA) to produce pyruvate and ATP [23]. The cofactor acetyl coenzyme A, generated from pyruvate by pyruvate dehydrogenase $(\mathrm{PDH})$, is required directly for the tricarboxylic acid (TCA) cycle and other biochemical reactions [24]. Citrate synthase (CS), aconitate hydratase (ACO), succinyl-CoA synthetase (SCS), 2-oxoglutarate dehydrogenase (OGDH), dihydrolipoyllysine-residue succinyltransferase (DLST), succinate dehydrogenase (SDH), fumarate hydratase (FUM), and malic dehydrogenase $(\mathrm{MDH})$ are also involved in the TCA cycle $[25,26]$.

Ascorbic acid (also named ascorbate or vitamin $\mathrm{C}$ ) is one of the ubiquitous watersoluble low molecular weight antioxidants in fruits, such as guava [5] and kiwifruit [27]. Ascorbic acid is essential for human health, intaking from edible plants due to humans lack of the last step ascorbate synthetic enzyme [28]. Four de novo biosynthesis pathways of ascorbic acid: the L-galactose, L-gulose, myo-inositol, and D-galacturonate pathways have been proposed in plants [29]. The L-galactose pathway of ascorbate biosynthesis starting from glucose has been extensively studied in higher plants [30]. Mannose-6-phosphate isomerase (PMI), phosphomannomutase (PMM), mannose-1-phosphate guanylyltransferase (GMP), GDP-mannose 3,5-epimerase (GME), GDP-L-galactose phosphorylase (GGP), L-galactose-1-phosphate phosphatase (GPP), L-galactose dehydrogenase (GDH), and Lgalactono-1,4-lactone dehydrogenase (GLDH) related to the ascorbate biosynthesis while L-ascorbate oxidase (AO), L-ascorbate peroxidase (APX), dehydroascorbate reductase (DHAR), and monodehydroascorbate reductase (MDAR) control the regeneration of ascorbate $[5,30]$. 
Elucidation of key metabolites and candidate genes responsible for fruit quality is beneficial for breeding new pitaya cultivars and improving their nutritional values. To date, candidate genes related to betalain biosynthesis were isolated from transcriptome data of red-peel and yellow-peel pitayas [31,32]. Besides, several candidate genes involved in sugar biosynthesis were also obtained through RNA-Seq [16]. However, compared with red-peel pitayas, genes related to sugars and organic acids in fruit development and maturation of yellow-peel pitaya are rarely studied. In this study, nine fruit development stages of 'WCHL' and 'YCHL' pitaya pulps were collected to investigate the changes in the total phenol, total flavonoid, ascorbate, starch, soluble sugar, major sugar, and organic acid components. Subsequently, the transcription abundance of candidate genes related to ascorbate synthesis and regeneration, starch synthesis and degradation, major sugar synthesis and TCA cycle were analyzed in the three fruit developmental stages of 'WCHL' and 'YCHL' pitaya pulps.

\section{Materials and Methods}

\subsection{Plant Materials}

Two yellow-peel pitayas i.e., Wucihuanglong (H. undatus, yellow peel with white flesh ('WCHL')) and Youcihuanglong (H. megalanthus, yellow peel with white flesh, ('YCHL')) were used as materials. The pulps of nine developmental stages (S1-S9) of 'WCHL' (14, 17, 19, 23, 25, 27, 29, 32, and 35 day after flowering (DAF)) and 'YCHL' (23, 35, 45, 55, 65, 70, 75, 85, and 96 DAF) (Figure 1) from different plants were used for analyses of sugar and organic acid. Pulps from three key fruit development stages of 'WCHL' (17, 25 and 29 DAF) and 'YCHL' (35, 65 and 75 DAF) pitaya were used for RNA-Seq with three biological repetitions (PRJNA797242). All samples were immediately frozen in liquid nitrogen and stored at $-80{ }^{\circ} \mathrm{C}$ until use.
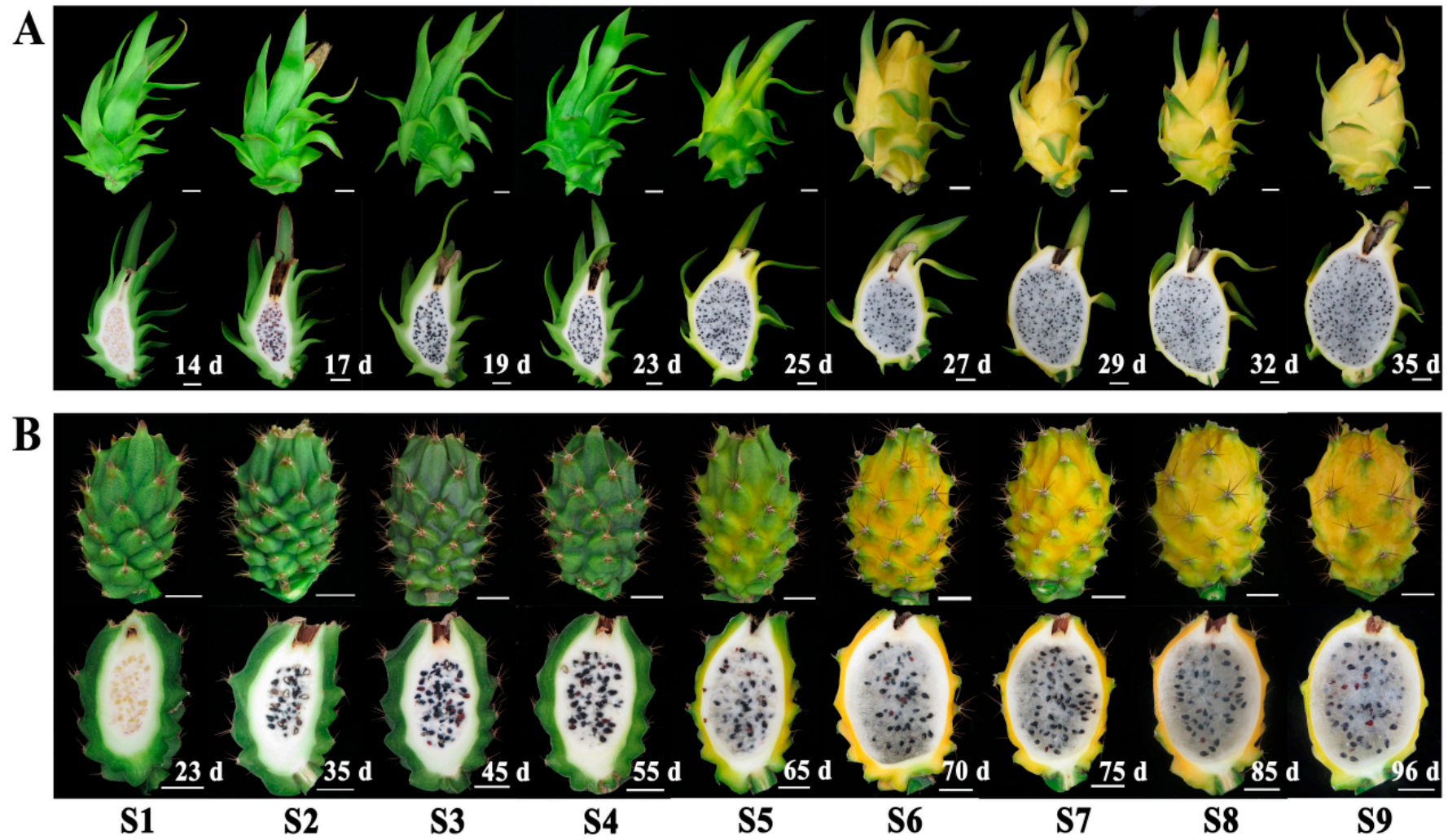

Figure 1. Photograph of 'WCHL' (A) and 'YCHL' (B) pitayas at nine (S1-S9) developmental stages. 


\subsection{Measurements of Total Phenols}

Total phenols were measured by the Folin-Ciocalteu method [33]. A total of $0.5 \mathrm{~g}$ samples were extracted with $10 \mathrm{~mL} 80 \%$ aqueous methanol $(v / v)$ solution. After centrifugation at $5000 \mathrm{rpm}$ for $10 \mathrm{~min}, 20 \mu \mathrm{L}$ supernatants with $1.8 \mathrm{~mL}$ foline-phenol $\left(0.2 \mathrm{~mol} \cdot \mathrm{L}^{-1}\right)$ were incubated at room temperature for $5 \mathrm{~min}$, and then incubated with $1.2 \mathrm{~mL} 15 \% \mathrm{Na}_{2}(\mathrm{CO})_{3}$ in $40^{\circ} \mathrm{C}$ for $30 \mathrm{~min}$ and measured at $760 \mathrm{~nm}$ through a spectrophotometer (Infinite M200, Tecan Co., Ltd., Shanghai, China).

\subsection{Measurements of Total Flavonoids}

The total flavonoids were measured by aluminum chloride [33]. A total of $0.5 \mathrm{~g}$ samples were extracted with $10 \mathrm{~mL} \mathrm{80 \%} \mathrm{aqueous} \mathrm{methanol}(v / v)$ solution. After centrifugation at $5000 \mathrm{rpm}$ for $10 \mathrm{~min}, 100 \mu \mathrm{L}$ supernatants, $1.9 \mathrm{~mL} \mathrm{90 \%} \mathrm{aqueous} \mathrm{ethanol}$

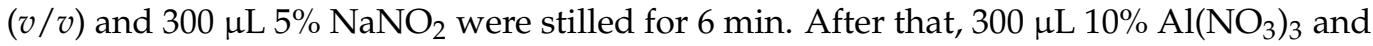
$2 \mathrm{~mL} 4 \% \mathrm{NaOH}$ were added to each mixture with an interval of $6 \mathrm{~min}$, and measured by spectrophotometer at $510 \mathrm{~nm}$ after standing $10 \mathrm{~min}$. All determinations were performed in three biological repetitions.

\subsection{Measurements of Starch and Soluble Sugar}

Samples of $0.5 \mathrm{~g}$ were extracted with $8 \mathrm{~mL} 80 \%$ aqueous methanol $(v / v)$ solution and put in a water bath at $80{ }^{\circ} \mathrm{C}$ for $30 \mathrm{~min}$. The supernatants were collected after centrifugation at $5000 \mathrm{rpm}$ for $10 \mathrm{~min}$. A total of $10 \mathrm{mg}$ activated carbon (AC) was added to the supernatants and then incubated in a water bath at $80^{\circ} \mathrm{C}$ for $30 \mathrm{~min}$. After filtrating the $\mathrm{AC}$, the supernatants were diluted to $25 \mathrm{~mL}$ with distilled water. The supernatants and residues were collected for measuring soluble sugar and starch, respectively.

A total of $2 \mathrm{~mL}$ distilled water was added to the residues (dried in $80^{\circ} \mathrm{C}$ ) and kept in boiling water for $10 \mathrm{~min}$. After cooling, $2 \mathrm{~mL} 9.2 \mathrm{~mol} \cdot \mathrm{L}^{-1}$ perchloric acid and $6 \mathrm{~mL}$ distilled water were added with an interval of $10 \mathrm{~min}$. The supernatants were collected by centrifuging at $5000 \mathrm{rpm}$ for $10 \mathrm{~min}$. The resides were subjected to a similar second extraction, and then the supernatants were collected for analyses of the absorbance values at $620 \mathrm{~nm}$ by a spectrophotometer.

A total of $1 \mathrm{~mL}$ supernatants with $5 \mathrm{~mL}$ anthrone $\left(1 \mu \mathrm{g} \cdot \mathrm{mL}^{-1}\right)$ were kept in boiling water for $10 \mathrm{~min}$, and then the absorbance values were measured at $620 \mathrm{~nm}$ by a spectrophotometer.

\subsection{Measurements of Sugars and Organic Acids}

A gas chromatography-mass spectrometry (GC-MS) was used to assay sugars and organic acids according to Lisec et al. [34] with minor modifications. Samples of $50 \mathrm{mg}$ were extracted with $1.4 \mathrm{~mL} 70 \%$ aqueous methanol $(v / v)\left(20{ }^{\circ} \mathrm{C}\right)$. Then, $60 \mu \mathrm{L}$ ribitol $\left(0.2 \mathrm{mg} \cdot \mathrm{mL}^{-1}\right.$, internal quantitative standard) was added to each mixture and followed by shaking in a Thermomixer Compact (Eppendorf, Germany) at $70{ }^{\circ} \mathrm{C}$ with the speed of $950 \mathrm{rpm}$ for $10 \mathrm{~min}$. The supernatants were collected by centrifugation at $11,000 \times g$ for $10 \mathrm{~min}$. A total of $750 \mu \mathrm{L}$ chloroform and $1.5 \mathrm{~mL}$ distilled water were added to the supernatants and then centrifuged at $2200 \times g$ for $15 \mathrm{~min}$. A total of $100 \mu \mathrm{L}$ of the upper phase (polar phase) was dried in a $1.5 \mathrm{~mL}$ tube by a vacuum concentrator (Eppendorf Concentrator plus, Germany) at room temperature. The dried samples were oximated with $20 \mu \mathrm{L}$ of methoxyamination reagent in $37^{\circ} \mathrm{C}$ for $2 \mathrm{~h}$ in a Thermomixer Compact (950 rpm) and the derivatization reaction without samples was used as the control. After oximation, silylation occurred by adding $35 \mu \mathrm{L}$ of MSTFA to each mixture and incubated in a Thermomixer Compact $(950 \mathrm{rpm})$ at $37^{\circ} \mathrm{C}$ for $30 \mathrm{~min}$. A total of $40 \mu \mathrm{L}$ of each derivatization product were transferred into glass vials for GC-MS analyses using an Agilent 7890A GC system equipped with an Agilent 7693 autosampler and Agilent 5975Cinert MSD with Triple Axis Detector (Agilent, Atlanta, GA, USA). The operating parameters were performed according to Hua et al. [3]. 


\subsection{RNA Extraction and Sequencing}

Three key fruit development stages (S2, S5, and S7) of 'WCHL' and 'YCHL' pitaya pulps were used for RNA-Seq with three biological repetitions (PRJNA797242). The total RNA was extracted using the RNA Prep Pure Plant Kit (TIANGEN, Beijing, China) according to the manufacturer's instructions. The integrity was assessed using the RNA Nano 6000 Assay Kit of the Bioanalyzer 2100 system (Agilent Technologies, Santa Clara, CA, USA). A total of $1 \mu \mathrm{g}$ total RNA of each sample was used as input material for the RNA sample preparations. The first-strand cDNA was synthesized using random hexamer primer and M-MuLV Reverse Transcriptase (RNase H-). The second-strand cDNA was synthesized using DNA Polymerase I and RNase $\mathrm{H}$. The library fragments were purified with AMPure XP system (Beckman Coulter, Beverly, MA, USA) to select cDNA fragments of preferentially 370 420 bp in length. PCR was performed with Phusion High-Fidelity DNA polymerase, Universal PCR primers and Index $(X)$ Primer. The library quality was assessed using the Agilent Bioanalyzer 2100 system (Agilent, Santa Clara, CA, USA). The clustering of the index-coded samples was performed on a cBot Cluster Generation System using TruSeq PE Cluster Kit v3-cBot-HS (Illumia, San Diego, CA, USA) according to the manufacturer's instructions. After cluster generation, the library preparations were sequenced on an Illumina Novaseq platform and $150 \mathrm{bp}$ paired-end reads were generated.

\subsection{Transcriptome Data Analyses}

Raw data (raw reads) of FASTQ format were firstly processed through in-house perl scripts. Clean data (clean reads) were obtained by removing reads containing adapter, reads containing ploy-N and low-quality reads from raw data. Q20, Q30, and GC content of the clean data were calculated and listed in Table S1. The datasets were functionally annotated by pitaya genome using Hisat2 (version 2.0.5) [35]. The quantification of each gene expression level (fragments per kilobase millions (FPKM)) was counted using featureCounts (version 1.5.0-p3). The differential expression analyses of two libraries were performed using the DESeq2 package (version 3.10) of R software (version 4.1.2). Kyoto Encyclopedia of Genes and Genomes (KEGG) enrichment analyses were performed using KofamKOALA (https:/ / www.genome.jp/tools/kofamkoala/, accessed on 5 February 2022). The transcript abundance of candidate genes was drawn by Tbtools software [36]. The accuracy of the RNA-Seq data was verified by RT-qPCR with specific primers (Table S2) according to the method of Xie et al. [32].

\subsection{Statistical Analysis}

Comparisons of the groups were performed by a one-way analysis of variance (Oneway ANOVA) with a Duncan test $(p<0.01)$. The correlation coefficients were calculated by the SPSS 25 software (SPSS Inc., Chicago, IL, USA) through Pearson's correlation and a two-tailed $t$ test $(p<0.05$ and $p<0.01)$.

\section{Results}

\subsection{Changes in Total Phenol, Total Flavonoid and Ascorbic Acid Contents during Fruit Development}

Phenol, flavonoid, and ascorbic acid belong to the bioactive compounds which play important roles in plant antioxidant activity [37]. The change in total phenol, total flavonoid, and ascorbic acid contents were analyzed during fruit development of 'WCHL' and 'YCHL' pitayas. Total phenol and flavonoid contents reached their maximum at stage 1 during fruit development of 'WCHL' and 'YCHL' pitayas (Figure 2A,B). Higher contents of total phenols and flavonoids were detected in 'WCHL' pitaya than that of 'YCHL' pitaya at ripening stages. Meanwhile, higher contents of ascorbic acid were detected in 'WCHL' pitaya than that of 'YCHL pitaya (Figure 2C). These data suggested that 'WCHL' pitaya accumulates more bioactive compounds than that of 'YCHL' pitaya. 

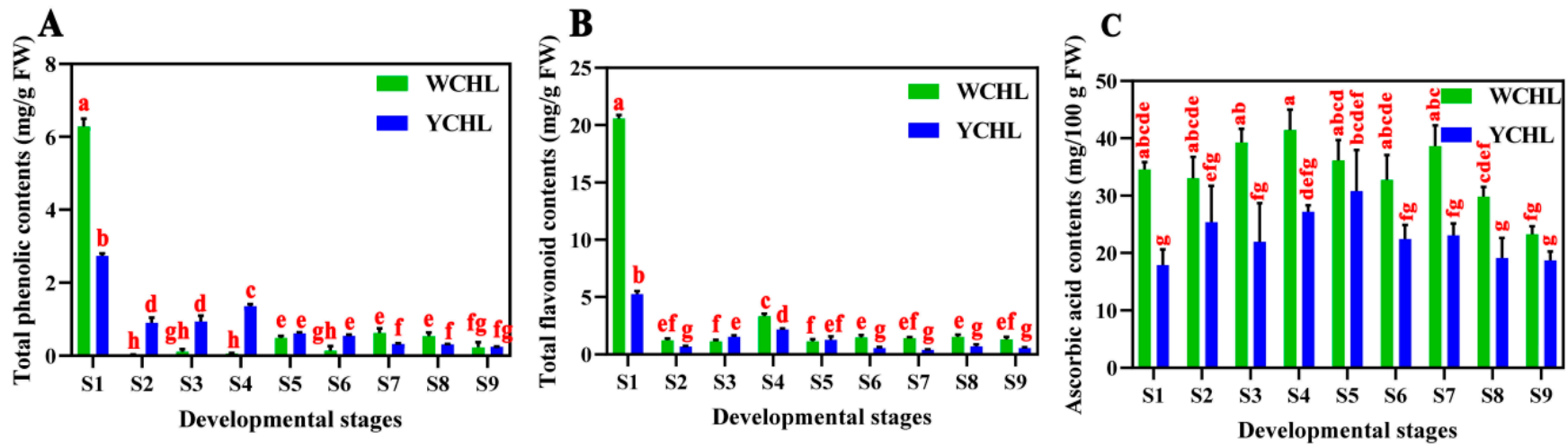

Figure 2. Changes in contents of total phenol (A), flavonoid (B), and ascorbic acid (C) in pulps during fruit developmental stages of 'WCHL' and 'YCHL' pitayas. Data represent the mean \pm S.E. of three biological replicates. Lowercase is indicated the comparison between groups using one-way ANOVA (Duncan test, $p<0.01$ ).

\subsection{Changes in Sugar Contents during Fruit Development}

Sugar contents were analyzed in pulps during fruit development of 'WCHL' and 'YCHL' pitayas. Starch showed an increasing pattern from stage 1 to stage 3, and declined from stage 3 to stage 5 and kept low levels thereafter (Figure 3A), suggesting that starch was synthesized from stage 1 to stage 3 and degraded after stage 3. Soluble sugar showed an upward trend and higher contents were detected in pulps of 'WCHL' pitaya than that of 'YCHL' pitayas during fruit development (Figure 3B). These results suggested that starch was mainly accumulated before stage 4 while soluble sugar was highly accumulated after stage 5, indicating that starch synthesis and degradation resulted in the accumulation of soluble sugars during fruit development of the two pitaya cultivars.

The sugar components including glucose, sucrose, fructose, galactose, inositol, and sorbitol were assayed during fruit development of the two yellow-peel pitayas (Figure 3C-H). Glucose, fructose, sorbitol, and galactose kept increasing tendency while sucrose and inositol began to highly accumulated after stage 5 during fruit maturation. Sucrose, fructose, and glucose were the dominant soluble sugars that highly accumulated at the later stages (from stage 5 to stage 9) in 'YCHL' pitayas (Figure 3J), while the main soluble sugar was glucose in 'WCHL' pitayas (Figure 3I). These results suggested that the sweetness of 'YCHL' pitaya is mainly determined by sucrose, fructose, and glucose compared with only glucose for 'WCHL' pitaya.

\subsection{Changes in Organic Acids during Pitaya Fruit Development}

The changes in six organic acids, i.e., oxalic, malic, fumaric, succinic, citric, and citramalic acids during fruit development of 'WCHL' and 'YCHL' pitayas were presented in Figure 4. Malic and citric acid contents were increasing until stage 4 and then gradually decreased during pitaya fruit development of 'WCHL' and 'YCHL' pitayas (Figure 4A,B). However, citramalic acids were mainly accumulated in the unripe stages and rapidly degraded in the ripe stages of 'WCHL' pitaya (Figure 4C). Higher contents of malic, citramalic and fumaric acid were detected in pulps of 'WCHL' pitaya than that of 'YCHL' pitaya while citric acid was higher accumulated in 'YCHL' pitaya than that of 'WCHL' pitaya (Figure 4A,C,E). Oxalic and succinic acids kept at lowly levels without significant difference between two pitayas (Figure 4D,F). Thus malic and citric acids were respectively the dominant organic acids in 'WCHL' and 'YCHL' pitayas, and contribute to sourness of pitaya pulps (Figure $4 \mathrm{G}, \mathrm{H}$ ). 

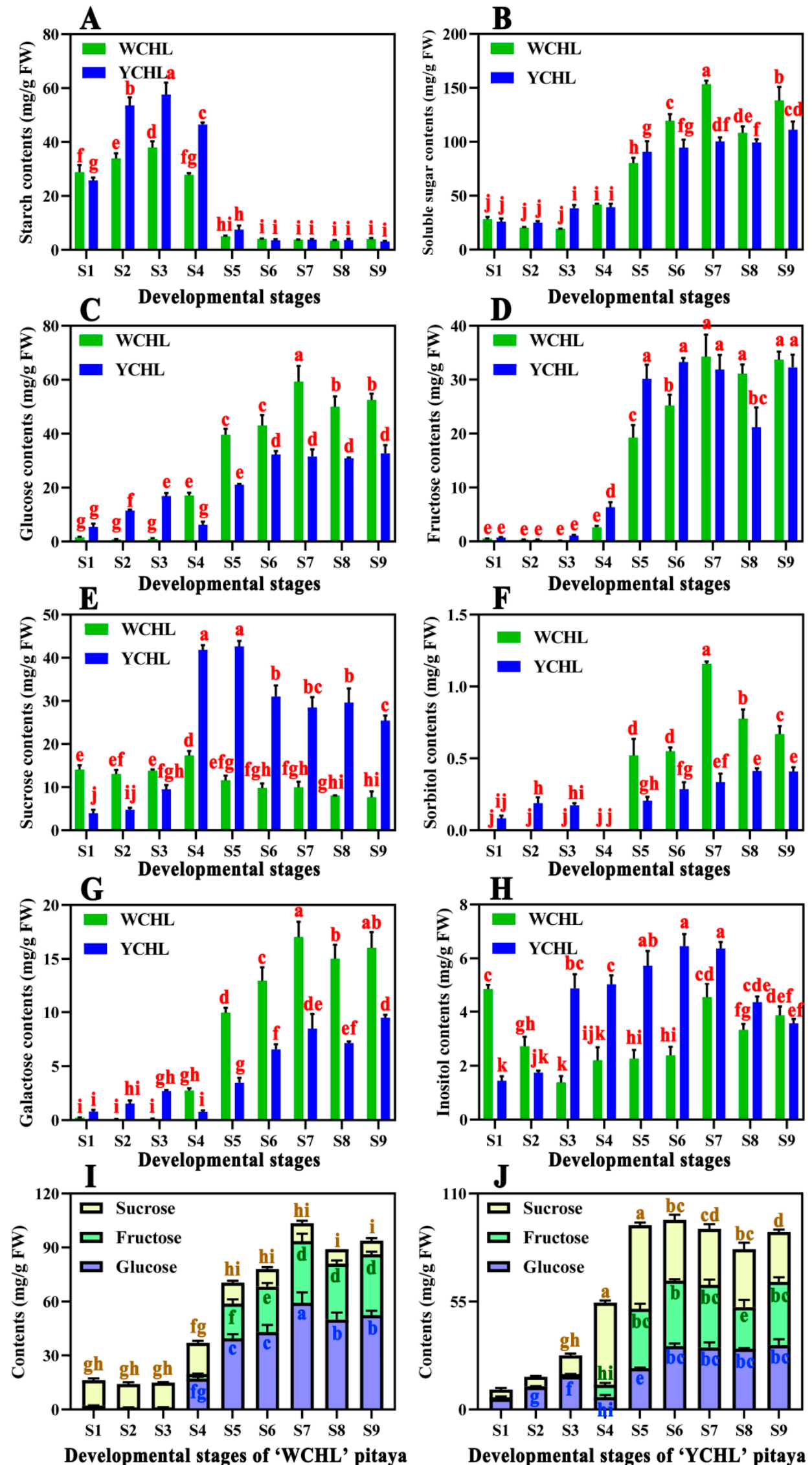

Figure 3. Changes in sugar contents in pulps during fruit developmental stages of 'WCHL' and 'YCHL' pitayas. (A) Starch contents; (B) soluble sugar contents; (C) glucose contents; (D) fructose 
contents; (E) sucrose contents; (F) sorbitol contents; (G) galactose contents; $(\mathbf{H})$ inositol contents; (I) major sugar contents in pulps during fruit developmental stages of 'WCHL' pitaya; (J) major sugar contents in pulps during fruit developmental stages of 'YCHL' pitaya. Data represent the mean \pm S.E. of three biological replicates. Lowercase indicates the comparison between groups using one-way ANOVA (Duncan test, $p<0.01$ ).

A

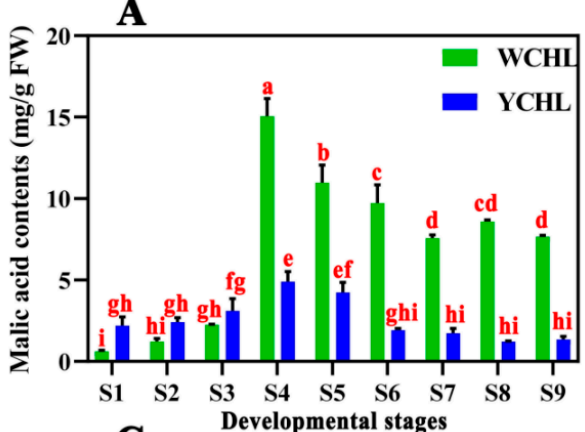

C
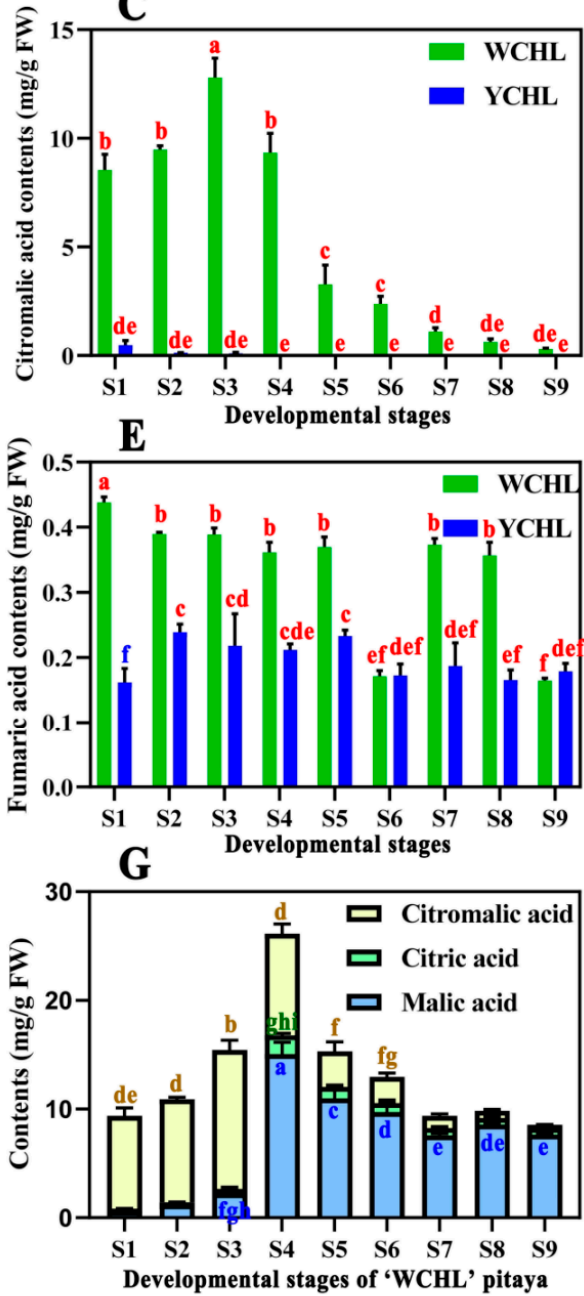

B

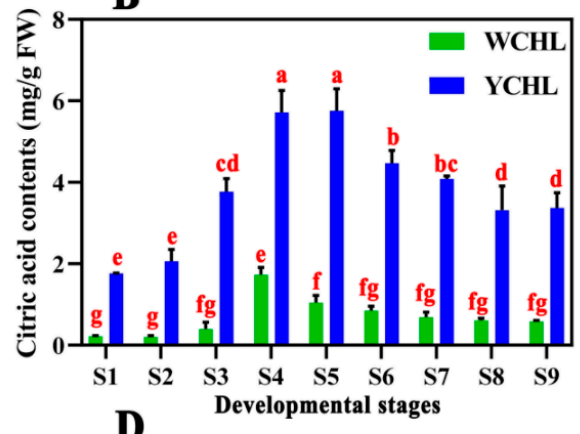

D
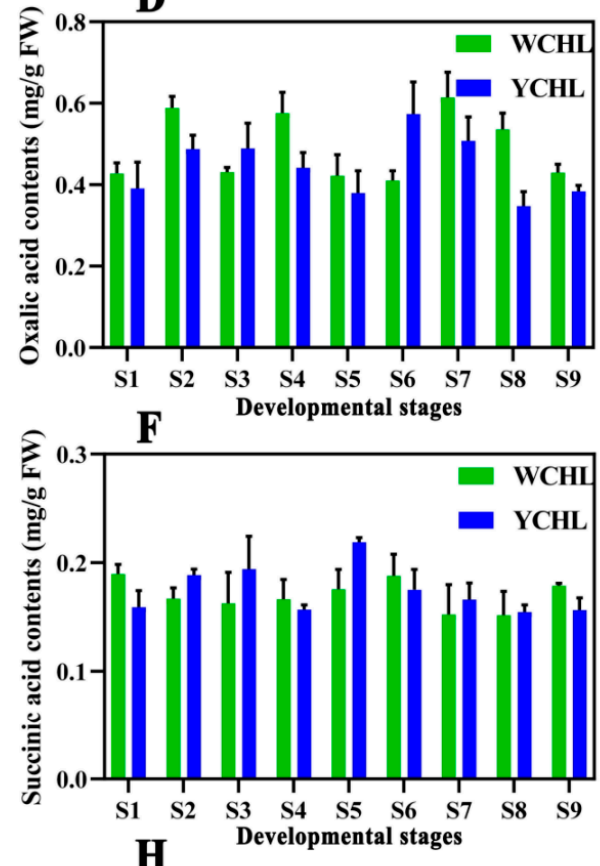

H

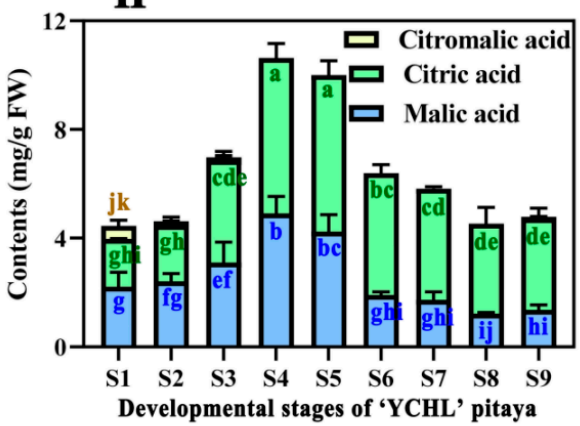

Figure 4. Changes in organic acid contents in pulps during fruit developmental stages of 'WCHL' and 'YCHL' pitayas. (A) Malic acid contents; (B) citric acid contents; (C) citromalic acid contents; (D) oxalic acid contents; (E) fumaric acid contents; (F) succinic acid contents; (G) main acid contents in pulps during fruit developmental stages of 'WCHL' pitaya; $(\mathbf{H})$ main acid contents in pulps during fruit developmental stages of 'YCHL' pitaya. Data represent the mean \pm S.E. of three biological replicates. Lowercase indicates the comparison between groups using one-way ANOVA (Duncan test, $p<0.01)$. 


\subsection{Analyses of Differentially Expressed Genes (DEGs)}

A total of $6.45 \mathrm{G}, 6.42 \mathrm{G}, 6.50 \mathrm{G}, 6.37 \mathrm{G}, 6.78 \mathrm{G}$, and $6.53 \mathrm{G}$ clean data were obtained from $17 \mathrm{~d}$ (S2), $25 \mathrm{~d}$ (S5), and $29 \mathrm{~d}$ (S7) of 'WCHL' and $35 \mathrm{~d}$ (S2), $65 \mathrm{~d}$ (S5), and $75 \mathrm{~d}$ (S7) of 'YCHL' pitaya pulps, respectively (Table S1). Based on the analyses of differentially expressed genes (DEGs) (padj $\leq$ 0.05), totally 10,420 and 9060 DEGs were respectively found in the three developmental stages of 'WCHL' and 'YCHL' pitaya pulps. Among them, most genes (9053 DEGs) were differentially expressed both in 'WCHL' and 'YCHL' pitaya pulps during fruit maturation (Figure 5A). A total of 4823 and 4111 DEGs were upregulated while 5597 and 4949 DEGs were down-regulated in 'WCHL' and 'YCHL' pitayas, respectively (Figure 5B). Moreover, most genes were enriched in the metabolic pathway, especially for secondary metabolites of sugar and acid, including carbon metabolism, glycolysis/gluconeogenesis, and starch and sugar metabolism (Figure 5C,D). Besides, the Pearson's correlation coefficient R2 between RNA-seq results and RT-qPCR results was 0.8078 , which was higher than the threshold of 0.7 , indicating that the FPKM values from RNA-Seq is reliable and can be used for subsequent experiments (Figure S1).
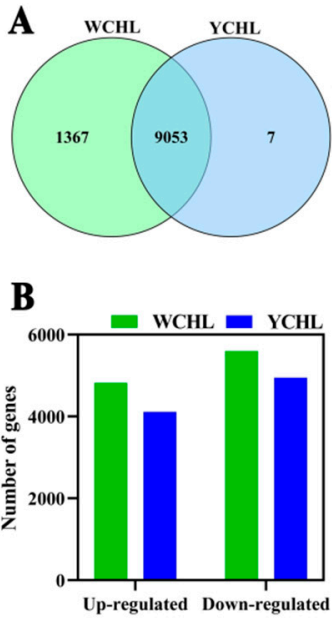

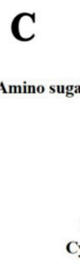

Cell cycle - yeast mRNA surveillance pathway ducleotide sugar metabolism $=$ Starch and sucrose metabolism
Purine metabolism Plant MAPK signaling pathway Plant MAPK signaling pathway
Ubiquitin mediated proteolysis Pyruvate metabolism $=$ Plant hormone signal transduction
ysteine and methionine metabolism Glycolysis/Gluconeogenesis Nucleocyloplasmictransport Ribosome biogenesis in eukaryotes
Thermogenesis Thermogenesis
Protein processing in endoplasmic reticulum Oxidative phosphorylation Spliceosome translation Ribosome
Biosynthesis of amino acids Biosynthesis of cofactors Carbon metabolism Carbon metabolism
diverse environments condary metabolites Metabolic pathways

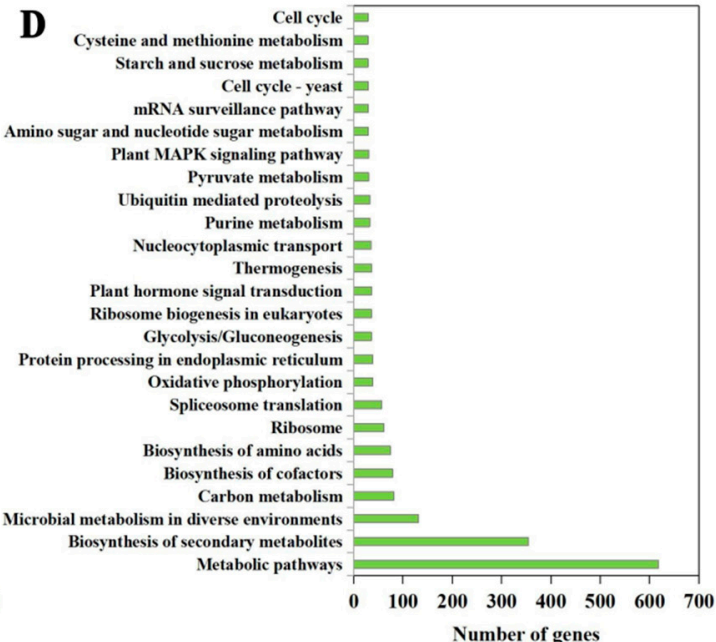

Figure 5. DEGs analyses of 'WCHL' and 'YCHL' pitaya pulps during three developmental stages. (A), Comparison of DEGs between 'WCHL' and 'YCHL' pitaya pulps; (B), the number of up- and down-regulated genes in 'WCHL' and 'YCHL' pitaya pulps; (C,D), the KEGG enrichment analysis of DEGs from 'WCHL' (C) and 'YCHL' (D) pitaya pulps.

\subsection{Candidate Genes Involved in Ascorbic Acid Metabolism}

Based on the ascorbic acid biosynthesis and regeneration pathway, candidate genes encoding its key enzymes were investigated in 'WCHL' and 'YCHL' pitayas. The Lgalactose, L-gulose, myo-inositol and D-galacturonate pathways were proposed to be involved in ascorbate biosynthesis in plants [5,38]. In the two yellow-peel pitayas, all enzymes associated with the L-galactose pathway were found and 61 candidate genes were obtained from the pathway (Table S3). Among these candidate genes, 27 genes were highly expressed in fruit development of yellow-peel pitayas, especially APX2 and MDAR3 that showed a downward trend in 'YCHL' pitayas and highly expressed at stage 5 in 'WCHL' pitaya (Figure 6; Table S3). According to the correlation analyses between the ascorbic acid contents and expression patterns of these candidate gene, PMM1, GMP3, GMP4, APX1, $A P X 9$, and MDAR1 were down-regulated during fruit development, and showed negative correlation relationship with ascorbic acid contents (Figure 6; Table S4). PMI1, PMI3 and $M D A R 2$ were up-regulated during fruit development and showed significant positive correlation relationship with ascorbic acid contents. These results indicated that PMI1 and $P M I 3$ are the potential key genes involved in ascorbic acid synthesis, while APXs (APX1, APX2, and APX9), and MDARs (MDAR1, MDAR2, and MDAR3) are responsible for ascorbic acid regeneration in 'WCHL' and 'YCHL' pitayas. 


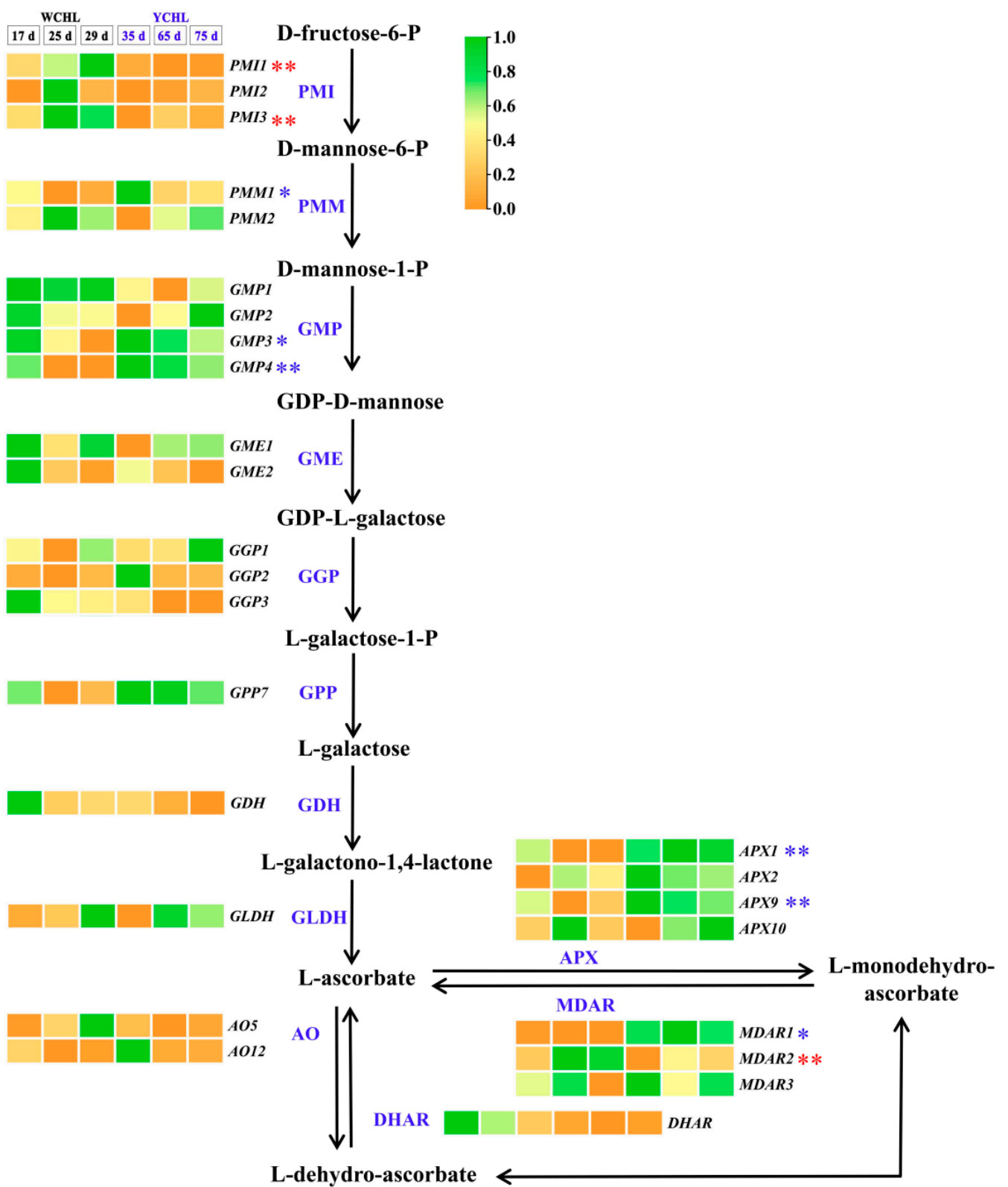

Figure 6. The transcript abundance of candidate genes involved in the proposed L-galactose pathway of ascorbate biosynthesis and regeneration during fruit maturation of 'WCHL' and 'YCHL' pitayas. Gene abbreviations are shown in Table S3. The correlation analyses of ascorbic acid contents and gene expressions are labeled as ${ }^{*}(p<0.05$, two-tailed $)$ or ${ }^{* *}(p<0.01$, two-tailed $)$, red indicates positive relationship while blue is negative.

\subsection{Candidate Genes Involved in Starch Metabolism}

The starch synthesis and degradation pathway has been proposed in plants [5]. A total of 90 candidate genes involved in starch synthesis and degradation were obtained in pitayas (Table S5). Among them, 32 candidate genes were highly expressed in pulps, especially five starch degradation related genes (AMY6, BAM10, BAM12, PHS2, and PHS3) which highly expressed at stage 5 during fruit development (Figure 7). A total of 13 candidate genes showed significantly negative correlation, including PGM2, StSy6, GWD2, GWD4, PWD1, BAM10, DPE1, AGL8, AMY6, AMY7, PHS2, PHS3, and PHS4 (Table S6). Additionally, the expression of AGPS2 and AGPS5 exhibited a downward trend and showed positive correlation with starch contents during fruit development. These data demonstrated that AGPSs (AGPS2 and AGPS5) are key genes related to the starch synthesis while AMY6, 
BAM10, PHS2, and PHS3 are important genes involved in starch degradation in pitaya (Table S6).
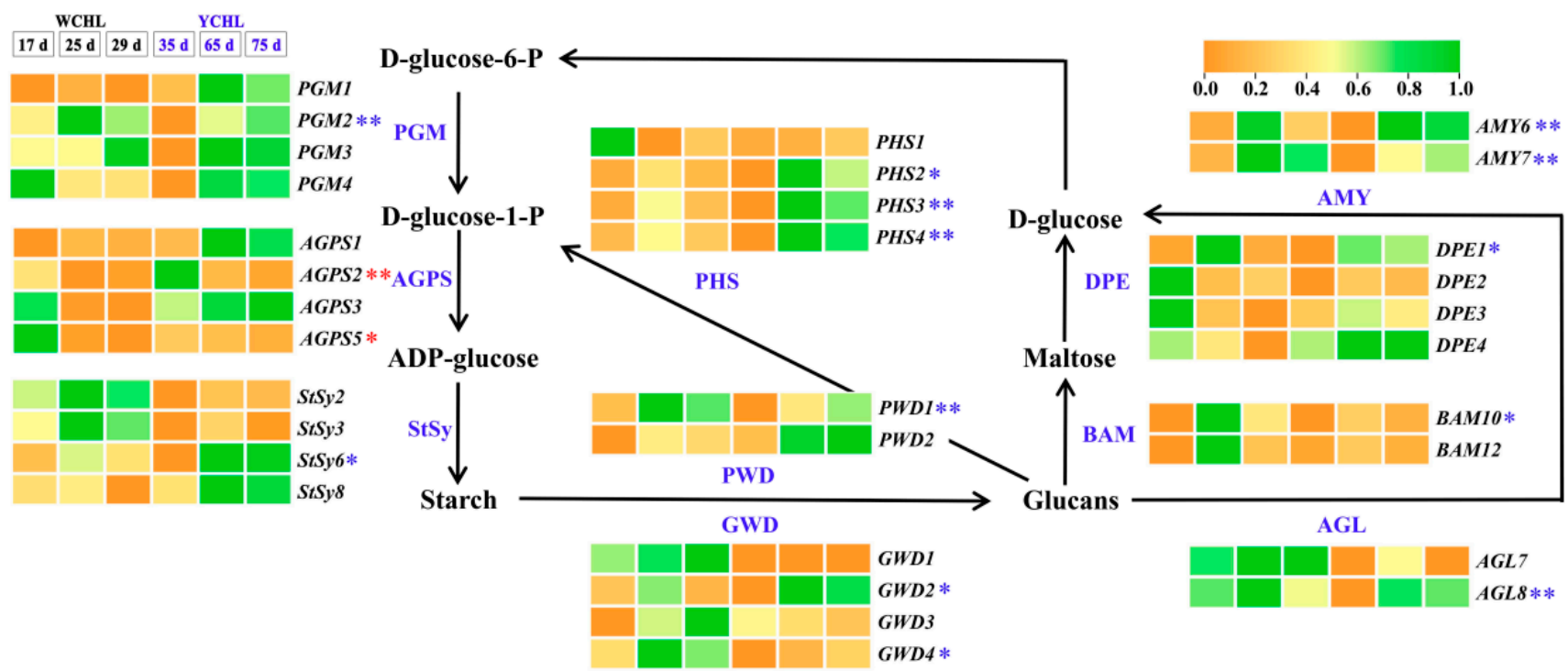

Figure 7. The transcript abundance of candidate genes involved in the proposed starch synthesis and degradation pathways during fruit maturation of 'WCHL' and 'YCHL' pitayas. Gene abbreviations are shown in Table S5. The correlation analyses of starch content and gene expressions are labeled as ${ }^{*}(p<0.05$, two-tailed $)$ or ${ }^{* *}(p<0.01$, two-tailed $)$, red indicates positive relationship while blue is negative.

\subsection{Candidate Genes Involved in Sugar Metabolism}

Totally, 73 candidate genes associated with sugar metabolism pathway were obtained in the transcriptome data, of which 18 candidate genes (three SPSs, four Ivrs, three SuSys, four HXKs, three FRKs, and one PGI) were highly expressed and probably coordinately regulate the sugar metabolism in the two yellow-peel pitayas (Table S7; Figure 8). Among them, higher expression levels of SuSy1, SuSy5, SuSy11, FRK5, FRK9, and FRK10 were detected, of which SuSy5, SuSy11, FRK5, and FRK9 showed a downward trend while SuSy1 and FRK10 were up-regulated during fruit development. The correlation analyses suggested that up-regulated genes, i.e., SPS1 and FRK9 showed positive correlation with sucrose and inositol contents while Ivr4 and HXK1 demonstrated positive correlation with glucose, fructose, sorbitol, and galactose accumulation (Figure 8; Table S8). SuSy5, SuSy11, Ivr10, FRK5, FRK10, HXK5, HXK9, and HXK12 had negative correlation with pitaya main sugars. These results suggested that SuSys (SuSy1, SuSy5 and SuSy11) and FRKs (FRK5, FRK9 and FRK10) are key genes to catalyze the pitaya sugar metabolism.

\subsection{Candidate Genes Associated with Organic Acids Metabolism}

The tricarboxylic acid (TCA) cycle was proposed to understand the major organic acids metabolism in yellow-peel pitayas (Figure 9). Totally 92 candidate genes were obtained and 41 genes were highly expressed in pulps (Table S9). The expressions of PEPC13, PEPCK, $A C O 1, A C O 2, D L S T 3, O G D H 3$, and SDH3 were increased and followed with decreasing during fruit development, consisting with the changes in malic acid contents (Table S10). CS2, CS12, Eno5, FUM1, MDH1, PEPC14, SCS1, and SCS2 maintained a decreasing expression pattern and showed negative correlation relationship with malic acid contents (Table S10). FUM1, OGDH2, PEPC3, and PEPC4 and SCS1 shared positive correlation relationship with citric acid contents, of which PEPC 3 and PEPC4 were highly expressed at stage 5 during fruit development with an extremely significant relationship (Table S10). Negative correlations were found between citric acid contents and the expression of PEPC13, PK9, PDH3, CMS1, CMS2, CMS3, DLST1, and OGDH3 with a higher expression level in 'WCHL' 
than 'YCHL' (Table S10). These positive and negative correlations with gene expressions were probably the main reason of more citric acid contents in 'YCHL' than 'WCHL'. In addition, only positive correlation relationships were detected between the citromalic acid contents and expression of down-regulated genes, including PGK4, Eno5, PK6, PK9, PDH3, PDH4, CMS1, CMS3, PEPC14, and CS1, CS2, CS4, CS6, CS10, and CS12 (Table S10). These results revealed that $P G K 4, E n o 5, P K 6, P K 9, P D H 3, P D H 4, P E P C 3$, PEPC4, PEPC13, PEPC14, and $P E P C K$ are key upstream genes which limited the carbon sources to enter the TCA cycle. CMS1, CMS2, and CMS3 are key genes involved in controlling the citromalic acid biosynthesis and competed acetyl-CoA with TCA cycle. Seventeen genes (CS1, CS2, CS4, CS6, CS10, CS12, ACO1, ACO2, SCS1, SCS2, OGDH2, OGDH3, DLST1, DLST3, SDH3, FUM1 and MDH1) are probably involved in organic acids metabolism of 'WCHL' and 'YCHL' pitayas.

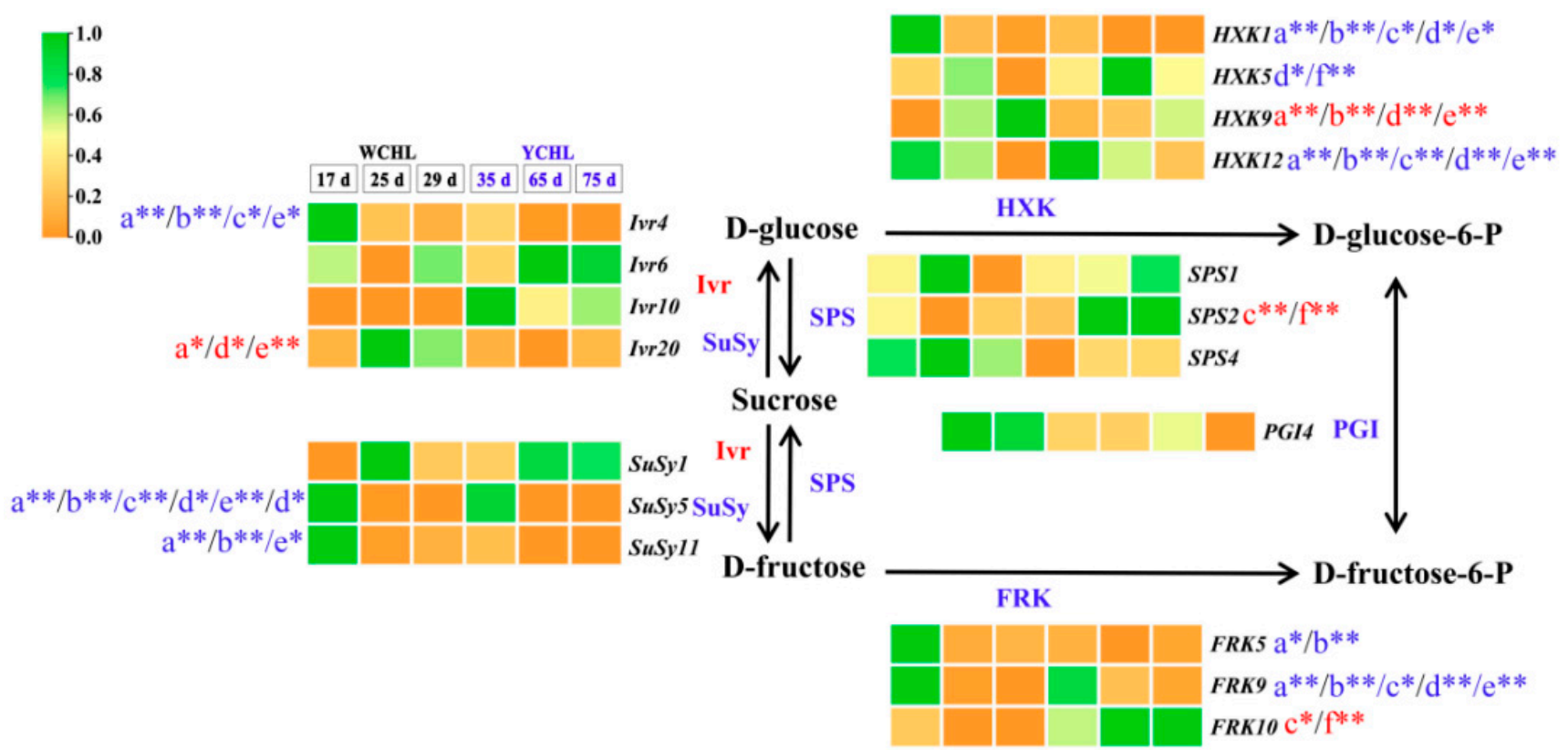

Figure 8. The proposed major sugar synthesis pathways and transcript abundance of their genes in 'WCHL' and 'YCHL' pitayas. Gene abbreviations are shown in Table S7. Letters a-f represent glucose, fructose, sucrose, sorbitol, galactose and inositol, respectively. The correlation analyses of main sugar contents and gene expressions are labeled as ${ }^{*}(p<0.05$, two-tailed $)$ or ${ }^{* *}(p<0.01$, two-tailed), red indicates positive relationship while blue is negative. 


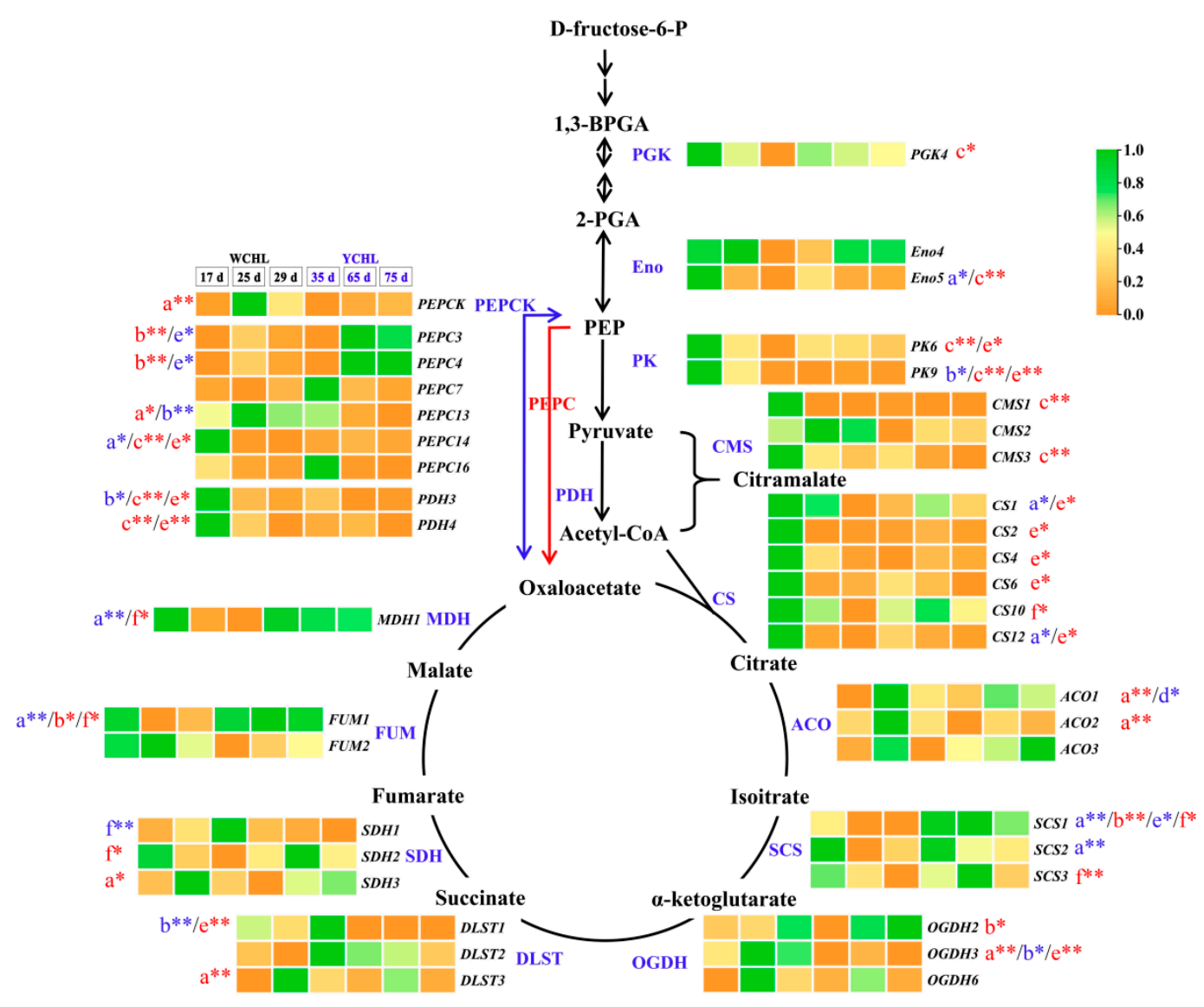

Figure 9. The proposed TCA cycle pathways in yellow pitaya and transcript abundance of their genes in 'WCHL' and 'YCHL' pitayas. Gene abbreviations are shown in Table S9. Letters a-f represent malic, citric, citromalic, oxalic acid, fumaric, and succinic acids, respectively. The correlation analyses of organic acid contents and gene expressions are labeled as $*(p<0.05$, two-tailed $)$ or $* *(p<0.01$, two-tailed), red indicates positive relationship while blue is negative.

\section{Discussions}

The metabolites and transcriptome profiling of red-peel and yellow-peel pitayas are used to elucidate a betalain biosynthesis pathway [3,32,39]. Metabolites, including phenol, flavonoid, starch, sugar and acid, are investigated in fruit development of redpeel pitayas [3,4]. The transcriptome analyses of red-peel pitayas showed that the key enzymes (invertase and sucrose synthase) and gene (HpVAI1) are involved in the sugar metabolism [16]. Those results provide fundamental information for developing pitaya quality and molecular breeding. However, little information is available about sugar and organic acid metabolism in yellow-peel pitayas. In this study, sugars and organic acids, and expression of metabolism-associated genes during fruit maturation of yellow-peel pitayas were analyzed. DEGs related to sugar and acid metabolism from RNA-Seq were verified by RT-qPCR (Pearson's correlation coefficient $\mathrm{R}^{2}=0.8078$; Figure S1).

Phenolics and flavonoids are the antioxidant compounds which can decrease the incidence of oxidative stress and associated with diseases in human health [33]. Phenolics and flavonoids are highly produced in unripe fruits [22] and mature fruits [40]. Besides, ascorbic acid is one of the most abundant antioxidants with various accumulation levels in different species [27,41]. In this study, higher contents of phenols and flavonoids were detected in pulps at the initial fruit development stages compared with low contents in mature stages (Figure 2A,B and Figure 10). A higher content of ascorbic acid was detected in 'WCHL' pitaya than that of 'YCHL' pitaya (Figure 2C). The L-galactose pathway represents the major route to L-ascorbic acid biosynthesis in higher plants. APX and MDHR are the key components involved in the ascorbate-glutathione cycle which operates in plant 
chloroplasts for $\mathrm{H}_{2} \mathrm{O}_{2}$ detoxifications [29,42]. In our study, 27 candidate genes involved in ascorbic acids synthesis and regeneration were obtained (Figure 6). APX2 and MDAR3 showed higher expression levels than the other genes during fruit development of yellowpeel pitayas (Table S4). Compared with the expression profiling and ascorbic acid contents in yellow-peel pitayas, MDAR2 were positively regulated the ascorbic acid contents while $A P X 1, A P X 9$, and MDAR1 showed negative correlation. These results indicate that APX and MDHR may play major roles in controlling the level of L-ascorbic acid in yellow-peel pitaya fruits and their gene family members were also identified and characterized in Arabidopsis thaliana [42,43].

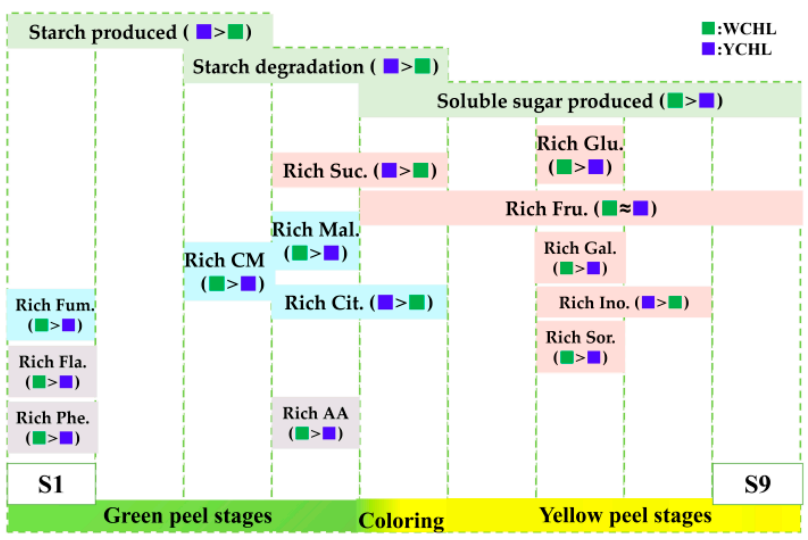

Figure 10. Schematic representation of sugar and organic acid metabolites during fruit developmental stages in two yellow-peel pitayas. Phe: phenolic; Fla: flavonoid; AA: ascorbic acid; Glu: glucose; Suc: sucrose; Fru: fructose; Gal: galactose; Ino: inositol; Sor: sorbitol; Mal: malic; Cit: citric; CM: citromalic; Fum: fumaric.

Carbohydrate was first synthesized as starch and then transferred into soluble sugars during fruit development of 'WCHL' and 'YCHL' pitayas (Figure 3A,B and Figure 10). The starch-to-sugar conversion is not only responsible for fruit sweetness but it also provides energy to coloration $[3,44]$. APS is a key enzyme of starch synthesis in seeds, tubers, and fruits [45-47]. In this work, 32 candidate genes (four PGMs, four AGPSs, four StSy, four GWDs, two PWDs, two AGLs, AMYs, two BAMs, four DPEs, and four PHSs) involved in starch synthesis and degradation were obtained (Figure 7). Expression patterns of most genes involved in starch degradation had a negative correlation with the changes in starch contents (Table S6). AGPS2 and AGPS5 kept a downward trend and shared a positive correlation with starch contents during fruit development of yellow-peel pitayas suggesting that AGPS is also a key enzyme for pitaya starch synthesis. AMY and BAM play a prominent role in starch breakdown and participate in the regulation of plant growth, development, and stress responses [48]. PHS participates in phosphorolytic degradation of starch [49]. In the present study, higher expression levels of AMY6, BAM10, PHS2, and PHS3 related to starch degradation pathways were detected than other genes and negative correlation with starch contents (Table S5), indicating that these genes probably play important roles in transferring starch into sugar in pitayas.

Sugars and organic acids have strong influence on fruit $\mathrm{pH}$, flavor, and taste [13]. Fructose, glucose, and sucrose were the main abundant sugar components reported in pitaya fruits $[3,4,16]$. In detail, the major sugar of 'WCHL' pitaya was glucose $(59.29 \mathrm{mg} / \mathrm{g}$ FW) (Figure 3), consistent with the results in red-peel pitayas [3]. However, the dominant sugars of 'YCHL' pitaya were sucrose (42.64 mg/g FW), fructose (33.28 mg/g FW), and glucose (32.72 mg/g FW). Most sugars are highly produced at S7 except sucrose which is rich in S4 and S5 of pitayas (Figure 10). The key enzymes, including SPS, SuSy, Ivr, HXK, and FXK, showed various expression patterns for regulating sugar contents during fruit developmental stages $[13,16]$. In our study, 18 candidate genes (three SPSs, three SuSys, four Ivrs, four HXKs, three FXKs, and one PGI) involved in sugar metabolism 
were obtained from yellow-peel pitayas (Figure 8). SPS2, Ivr20, FRK10, and HXK9 had a positive correlation while SuSy5, SuSy11, Ivr4, FRK5, FRK9, HXK1, HXK5 and HXK12 were negatively correlated with sugar accumulation during fruit development of yellowpeel pitayas (Figure 8; Table S8), suggesting that these genes cooperatively regulate sugar synthesis of yellow-peel pitayas.

Malic, citric, and citramalate acids were the three organic acids mainly accumulated at S2 to S5 in pitayas (Figure 10). Due to the degradation of citramalate acid in maturation stages, malic acid (15.06 mg/g FW) and citric acid (5.76 mg/g FW) were the most abundant organic acids in 'WCHL' and 'YCHL' pitayas, respectively. CMS has been reported as a key enzyme responsible for citramalate synthesis in Escherichia coli and apple [50,51]. In yellow-peel pitayas, the expression patterns of CMS1 and CMS3 had positive correlation with citromalic acid contents, indicating they probably responsible for citromalic acid biosynthesis of pitaya fruits. PEPC catalyzes the irreversible carboxylation of phosphoenolpyruvate (PEP) to form oxaloacetate which is the substrate for forming citrate $[14,52]$. In this study, the accumulation of citric acids was significant positive correlation with the expression of PEPC3 and PEPC4 during pitaya fruit development. Furthermore, CS catalyzes the reaction of oxaloacetate and acetyl-CoA convert into citrate and coenzyme A, which is the first step in the TCA cycle [52]. However, no CSs show correlated relationship with citrate in the two yellow-peel pitayas. This suggests that carbon fluxed into TCA cycle mainly via PEPC3 and PEPC4 rather than CSs, probably because rich acetyl-CoA fluxed into the irreversible step catalyzed by $C M S$ s for citramalate acid biosynthesis. MDH catalyzes the conversion between oxaloacetate and malate and FUM catalyzes the reversible interconversion between malate and fumarate [53,54]. A higher content of malic acid in 'WCHL' pitaya were detected than that of 'YCHL' pitaya. Malic acid content had a negative correlation with FUM1 and MDH1. Compared with 'YCHL' pitaya, MDH1 was strongly down-regulated in 'WCHL' pitaya responsible for more malic acid in 'WCHL' pitaya than 'YCHL' pitaya. Further work such as enzyme activity analyses and genetic transformation are necessary to elucidate their roles in modulating the fruit quality of pitayas.

\section{Conclusions}

In this study, higher total phenol and flavonoid contents were detected in the stage 1 of yellow-peel pitaya pulps during fruit development. A higher content of ascorbic acid was detected in 'WCHL' pitaya than 'YCHL' pitaya. Starch was mainly accumulated at early stages and transferred to soluble sugars at S5. Six sugars components (glucose, sucrose, fructose, galactose, inositol, and sorbitol) and six organic acids (oxalic, malic, fumaric, glyceric, succinic, citric and citromalic acid) were detected in pitayas. Glucose and malic acid were the key factors contributed to the taste and flavor quality of 'WCHL' pitaya. Sucrose, fructose, and glucose were the main sugars, and citric acid was the dominant acid responsible for fruit quality of 'YCHL' pitaya. Based on the expression profilings, 27 candidate genes probably involved in ascorbate biosynthesis and regeneration were achieved, of which PMIs was vital for ascorbic acid synthesis, and APXs and MDHRs played important roles in ascorbate accumulation. A total of 32 candidate genes probably involved in starch synthesis and degradation pathway were isolated, of which AGPSs were key genes for starch synthesis and $A M Y, B A M$ and $P H S$ s were key regulators controlling starch degradation. SPSs Ivrs, SuSys, HXKs, FRKs and PGI coordinately regulated sucrose, fructose, and glucose synthesis, of which SuSys and FRKs were expressed more than the other genes. Organic acids were lowly accumulated in ripening stages associated with downward expression patterns of PGK, Enos, PEPCs, PKs, and PDHs which limited the carbon flux into TCA cycle. Twenty candidate genes involved in organic acid production and degradation (TCA cycle) were obtained in pitayas. The findings of this study provide basic information for improving fruit quality of yellow-peel pitaya. 


\begin{abstract}
Supplementary Materials: The following supporting information can be downloaded at https:/ / www.mdpi.com/article/10.3390/plants11050694/s1, Figure S1: The RT-qPCR analyses of eleven candidate genes $(\mathrm{A}-\mathrm{K})$ in sugar and acid metabolic pathway and the coefficient analyses between FPKM values and RT-qPCR of those genes (L); Table S1: RNA-Seq data and corresponding quality control information; Table S2: Specific primers used in RT-qPCR assay; Table S3: The transcript abundance of genes related to ascorbate pathways in 'WCHL' and 'YCHL' pitayas pulps; Table S4: Correlation analyses between the ascorbic acid contents and expression patterns of candidate genes in 'WCHL' and 'YCHL' pitayas; Table S5: The transcript abundance of genes related to starch synthesis and degradation pathways in 'WCHL' and 'YCHL' pitayas; Table S6: Correlation analyses between the starch contents or total soluble sugar and expression patterns of candidate genes in 'WCHL' and 'YCHL' pitayas; Table S7: The transcript abundance of genes related to major sugar biosynthesis pathways in 'WCHL' and 'YCHL' pitayas; Table S8: Correlation analyses between the main sugar contents and expression patterns of candidate gene in 'WCHL' and 'YCHL' pitayas; Table S9: The transcript abundance of genes related to TCA cycle pathways in 'WCHL' and 'YCHL' pitayas; Table S10: Correlation analyses between the main organic acid contents and expression patterns of candidate genes in 'WCHL' and 'YCHL' pitayas.
\end{abstract}

Author Contributions: Conceptualization, F.X, J.C. (Jianye Chen) and Y.Q.; Methodology and validation, F.X and C.C.; Formal analysis, investigation, resources, data curation and visualization, F.X., C.C, J.C. (Jiaxuan Chen), Y.Y., Q.H., Z.Z., J.Z. and G.H.; Writing-original draft preparation, F.X.; Supervision, J.C. (Jianye Chen) and Y.Q.; Project administration, Y.Q.; Funding acquisition, J.C. (Jianye Chen) and Y.Q. All authors have read and agreed to the published version of the manuscript.

Funding: This research was funded by the Key Science and Technology Planning Project of Guangzhou (201904020015), Science and Technology Program of Guangzhou (202002020060) and Zhanjiang (2019A01003).

Institutional Review Board Statement: Not applicable.

Informed Consent Statement: Not applicable.

Data Availability Statement: Data is contained within the article.

Conflicts of Interest: The authors declare no conflict of interest.

\title{
References
}

1. Ibrahim, S.R.M.; Mohamed, G.A.; Khedr, A.I.M.; Zayed, M.F.; El-Kholy, A.A.S. Genus Hylocereus: Beneficial phytochemicals, nutritional importance, and biological relevance-A review. J. Food Biochem. 2018, 42, e12491. [CrossRef]

2. Suh, D.H.; Lee, S.; Heo, D.Y.; Kim, Y.; Cho, S.K.; Lee, C.H. Metabolite profiling of red and white pitayas (Hylocereus polyrhizus and Hylocereus undatus) for comparing betalain biosynthesis and antioxidant activity. J. Agric. Food Chem. 2014, 62, 8764-8771. [CrossRef] [PubMed]

3. Hua, Q.; Chen, C.; Tel, Z.N.; Wang, H.; Wu, J.; Noemi, T.Z.; Chen, J.; Zhang, Z.; Zhao, J.; Hu, G.; et al. Metabolomic characterization of pitaya fruit from three red-skinned cultivars with different pulp colors. Plant Physiol. Biochem. 2018, 126, 117-125. [CrossRef] [PubMed]

4. Wu, Y.; Xu, J.; He, Y.; Shi, M.; Han, X.; Li, W.; Zhang, X.; Wen, X. Metabolic profiling of pitaya (Hylocereus polyrhizus) during fruit development and maturation. Molecules 2019, 24, 1114. [CrossRef] [PubMed]

5. Chen, F.; Chao, F.; Lin, X.; Liu, S.; Li, Y.; Kang, M. A chromosome-level genome assembly provides insights into ascorbic acid accumulation and fruit softening in guava (Psidium guajava). Plant Biotechnol. J. 2021, 19, 717-730.

6. Abbas, H.M.K.; Huang, H.; Yang, Y.; Xie, Y.; Zou, J.; Xue, S.; Song, D.; Wu, T.; Li, J.; Zhong, Y. Characterization of starch in Cucurbita moschata germplasms throughout fruit development. J. Agric. Food Chem. 2020, 68, 9690-9696. [CrossRef] [PubMed]

7. Mahlow, S.; Orzechowski, S.; Fettke, J. Starch phosphorylation: Insights and perspectives. Cell. Mol. Life Sci. 2016, 73, $2753-2764$. [CrossRef]

8. Solis-Badillo, E.; Agama-Acevedo, E.; Tiessen, A.; Lopez Valenzuela, J.A.; Bello-Perez, L.A. ADP-Glucose pyrophosphorylase is located in the plastid and cytosol in the pulp of tropical banana fruit (Musa acuminata). Plant Foods Hum. Nutr. 2020, 75, 76-82 [CrossRef]

9. Prathap, V.; Tyagi, A. Correlation between expression and activity of ADP glucose pyrophosphorylase and starch synthase and their role in starch accumulation during grain filling under drought stress in rice. Plant Physiol. Biochem. 2020, 157, $239-243$.

10. Li, S.; Wang, T.; Guo, J.; Dong, Y.; Wang, Z.; Gong, L.; Li, X. Polystyrene microplastics disturb the redox homeostasis, carbohydrate metabolism and phytohormone regulatory network in barley. J. Hazard. Mater. 2021, 415, 125614. [CrossRef]

11. Li, L.; Tan, W.; Li, W.; Zhu, Y.; Cheng, Y.; Ni, H. Citrus taste modification potentials by genetic engineering. Int. J. Mol. Sci. 2019, 20, 6194. [CrossRef] [PubMed] 
12. Mayuoni-Kirshinbaum, L.; Porat, R. The flavor of pomegranate fruit: A review. J. Sci. Food Agric. 2014, 94, 21-27. [CrossRef] [PubMed]

13. Umer, M.J.; Bin Safdar, L.; Gebremeskel, H.; Zhao, S.; Yuan, P.; Zhu, H.; Kaseb, M.O.; Anees, M.; Lu, X.; He, N.; et al. Identification of key gene networks controlling organic acid and sugar metabolism during watermelon fruit development by integrating metabolic phenotypes and gene expression profiles. Hortic. Res. 2020, 7, 193. [CrossRef]

14. Zhang, Q.; Feng, C.; Li, W.; Qu, Z.; Zeng, M.; Xi, W. Transcriptional regulatory networks controlling taste and aroma quality of apricot (Prunus armeniaca L.) fruit during ripening. BMC Genom. 2019, 20, 45. [CrossRef] [PubMed]

15. Oikawa, A.; Otsuka, T.; Nakabayashi, R.; Jikumaru, Y.; Isuzugawa, K.; Murayama, H.; Saito, K.; Shiratake, K. Metabolic profiling of developing pear fruits reveals dynamic variation in primary and secondary metabolites, including plant hormones. PLoS ONE 2015, 10, e131408. [CrossRef]

16. Zhang, Z.; Xing, Y.; Ramakrishnan, M.; Chen, C.; Xie, F.; Hua, Q.; Chen, J.; Zhang, R.; Zhao, J.; Hu, G.; et al. Transcriptomics-based identification and characterization of genes related to sugar metabolism in 'Hongshuijing pitaya'. Hortic. Plant J. 2021. [CrossRef]

17. Ruan, Y.L. Sucrose metabolism: Gateway to diverse carbon use and sugar signaling. Annu. Rev. Plant Biol. 2014, 65, 33-67. [CrossRef]

18. Stein, O.; Granot, D. An overview of sucrose synthases in plants. Front. Plant Sci. 2019, 10, 95. [CrossRef]

19. Granot, D.; Kelly, G.; Stein, O.; David-Schwartz, R. Substantial roles of hexokinase and fructokinase in the effects of sugars on plant physiology and development. J. Exp. Bot. 2014, 65, 809-819. [CrossRef]

20. Lee, J.H.; Chang, K.Z.; Patel, V.; Jeffery, C.J. Crystal structure of rabbit phosphoglucose isomerase complexed with its substratedfructose 6-phosphate. Biochemistry 2001, 40, 7799-7805. [CrossRef]

21. Zhao, J.; Shen, F.; Gao, Y.; Wang, D.; Wang, K. Parallel bud mutation sequencing reveals that fruit sugar and acid metabolism potentially influence stress in Malus. Int. J. Mol. Sci. 2019, 20, 5988. [CrossRef] [PubMed]

22. Liu, Y.; Wu, Y.; Che, F.; Zhang, Z.; Chen, B. Physical-chemical composition and quality related changes in "ruaner" pear (Pyrus ussuriensis) during freezing-thawing period. Molecules 2019, 24, 2611. [CrossRef] [PubMed]

23. Ronimus, R.S.; Morgan, H.W. Distribution and phylogenies of enzymes of the Embden-Meyerhof-Parnas pathway from archaea and hyperthermophilic bacteria support a gluconeogenic origin of metabolism. Archaea 2003, 1, 199-221. [CrossRef] [PubMed]

24. Feng, Y.; Zhang, L.; Fu, J.; Li, F.; Wang, L.; Tan, X.; Mo, W.; Cao, H. Characterization of glycolytic pathway genes using RNA-Seq in developing kernels of Eucommia ulmoides. J. Agric. Food Chem. 2016, 64, 3712-3731. [CrossRef]

25. Dumont, S.; Rivoal, J. Consequences of oxidative stress on plant glycolytic and respiratory metabolism. Front. Plant Sci. 2019, 10, 166. [CrossRef]

26. Shen, Y.; Li, J.; He, F.; Zhu, J.; Han, Q.; Zhan, X.; Xing, B. Phenanthrene-triggered tricarboxylic acid cycle response in wheat leaf. Sci. Total Environ. 2019, 665, 107-112. [CrossRef]

27. Bulley, S.M.; Rassam, M.; Hoser, D.; Otto, W.; Schünemann, N.; Wright, M.; MacRae, E.; Gleave, A.; Laing, W. Gene expression studies in kiwifruit and gene over-expression in Arabidopsis indicates that GDP-L-galactose guanyltransferase is a major control point of vitamin C biosynthesis. J. Exp. Bot. 2009, 60, 765-778. [CrossRef]

28. Smirnoff, N. Ascorbic acid: Metabolism and functions of a multi-facetted molecule. Curr. Opin. Plant Biol. 2000, 3, 229-235. [CrossRef]

29. Cruz-Rus, E.; Amaya, I.; Valpuesta, V. The challenge of increasing vitamin C content in plant foods. Biotechnol. J. 2012, 7, 1110-1121. [CrossRef]

30. Bulley, S.; Laing, W. The regulation of ascorbate biosynthesis. Curr. Opin. Plant Biol. 2016, 33, 15-22. [CrossRef]

31. Hua, Q.; Chen, C.; Chen, Z.; Chen, P.; Ma, Y.; Wu, J.; Zheng, J.; Hu, G.; Zhao, J.; Qin, Y. Transcriptomic analysis reveals key genes related to betalain biosynthesis in pulp coloration of Hylocereus polyrhizus. Front. Plant Sci. 2016, 6, 1179.

32. Xie, F.; Hua, Q.; Chen, C.; Zhang, L.; Zhang, Z.; Chen, J.; Zhang, R.; Zhao, J.; Hu, G.; Zhao, J.; et al. Transcriptomics-based identification and characterization of glucosyltransferases involved in betalain biosynthesis in Hylocereus megalanthus. Plant Physiol. Biochem. 2020, 152, 112-124. [CrossRef] [PubMed]

33. Zhang, L.; Ravipati, A.S.; Koyyalamudi, S.R.; Jeong, S.C.; Reddy, N.; Smith, P.T.; Bartlett, J.; Shanmugam, K.; Münch, G.; Wu, M.J. Antioxidant and anti-inflammatory activities of selected medicinal plants containing phenolic and flavonoid compounds. J. Agric. Food Chem. 2011, 59, 12361-12367. [CrossRef]

34. Lisec, J.; Schauer, N.; Kopka, J.; Willmitzer, L.; Fernie, A.R. Corrigendum: Gas chromatography mass spectrometry-based metabolite profiling in plants. Nat. Protoc. 2015, 10, 1457. [CrossRef] [PubMed]

35. Chen, J.; Xie, F.; Cui, Y.; Chen, C.; Lu, W.; Hu, X.; Hua, Q.; Zhao, J.; Wu, Z.; Gao, D.; et al. A chromosome-scale genome sequence of pitaya (Hylocereus undatus) provides novel insights into the genome evolution and regulation of betalain biosynthesis. Hortic. Res. 2021, 8, 164. [CrossRef] [PubMed]

36. Chen, C.; Chen, H.; Zhang, Y.; Thomas, H.R.; Frank, M.H.; He, Y.; Xia, R. TBtools: An integrative toolkit developed for interactive analyses of big biological data. Mol. Plant 2020, 13, 1194-1202. [CrossRef] [PubMed]

37. Skrovankova, S.; Sumczynski, D.; Mlcek, J.; Jurikova, T.; Sochor, J. Bioactive compounds and antioxidant activity in different types of berries. Int. J. Mol. Sci. 2015, 16, 24673-24706. [CrossRef]

38. Aboobucker, S.; Suza, W.; Lorence, A. Characterization of two Arabidopsis L-Gulono-1,4-lactone oxidases, AtGulLO 3 and AtGulLO $_{5}$, involved in ascorbate biosynthesis. React. Oxyg. Species 2017, 4, 389-417. [CrossRef] 
39. Zhou, Z.; Gao, H.; Ming, J.; Ding, Z.; Lin, X.; Zhan, R. Combined transcriptome and metabolome analysis of pitaya fruit unveiled the mechanisms underlying peel and pulp color formation. BMC Genom. 2020, 21, 734. [CrossRef]

40. Cai, J.; Chen, T.; Zhang, Z.; Li, B.; Qin, G.; Tian, S. Metabolic dynamics during loquat fruit ripening and postharvest technologies. Front. Plant Sci. 2019, 10, 619. [CrossRef]

41. Mellidou, I.; Kanellis, A.K. Genetic control of ascorbic acid biosynthesis and recycling in horticultural crops. Front. Chem. 2017, 5, 50. [CrossRef] [PubMed]

42. Chew, O.; Whelan, J.; Millar, A.H. Molecular definition of the ascorbate-glutathione cycle in Arabidopsis mitochondria reveals dual targeting of antioxidant defenses in plants. J. Biol. Chem. 2003, 278, 46869-46877. [CrossRef] [PubMed]

43. Leterrier, M.; Corpas, F.J.; Barroso, J.B.; Sandalio, L.M.; Del Río, L.A. Peroxisomal monodehydroascorbate reductase. Genomic clone characterization and functional analysis under environmental stress conditions. Plant Physiol. 2005, 138, 2111-2123. [CrossRef] [PubMed]

44. Cordenunsi-Lysenko, B.R.; Nascimento, J.R.O.; Castro-Alves, V.C.; Purgatto, E.; Fabi, J.P.; Peroni-Okyta, F.H.G. The starch is (Not) just another brick in the wall: The primary metabolism of sugars during banana ripening. Front. Plant Sci. 2019, 10, 391. [CrossRef]

45. Miao, H.; Sun, P.; Liu, Q.; Liu, J.; Xu, B.; Jin, Z. The AGPase family proteins in banana: Genome-wide identification, phylogeny, and expression analyses reveal their involvement in the development, ripening, and Abiotic/Biotic stress responses. Int. J. Mol. Sci. 2017, 18, 1581. [CrossRef]

46. Ferrero, D.; Asencion, D.M.; Kuhn, M.L.; Falaschetti, C.A.; Piattoni, C.V.; Iglesias, A.A.; Ballicora, M.A. On the roles of wheat endosperm ADP-Glucose pyrophosphorylase subunits. Front. Plant Sci. 2018, 9, 1498. [CrossRef]

47. Ma, P.; Chen, X.; Liu, C.; Xia, Z.; Song, Y.; Zeng, C.; Li, Y.; Wang, W. MePHD1 as a PHD-Finger protein negatively regulates ADP-Glucose pyrophosphorylase small subunit1a gene in cassava. Int. J. Mol. Sci. 2018, 19, 2831. [CrossRef]

48. Chuan, Y.; Cao, H.; Lin, H.; Hu, J.; Ye, Y.; Li, J.; Hao, Z.; Hao, X.; Sun, Y.; Yang, Y.; et al. Expression patterns of alpha-amylase and beta-amylase genes provide insights into the molecular mechanisms underlying the responses of tea plants (Camellia sinensis) to stress and postharvest processing treatments. Planta 2019, 250, 281-298.

49. Rathore, R.S.; Garg, N.; Garg, S.; Kumar, A. Starch phosphorylase: Role in starch metabolism and biotechnological applications. Crit. Rev. Biotechnol. 2009, 29, 214-224. [CrossRef]

50. Wu, X.; Eiteman, M.A. Production of citramalate by metabolically engineered Escherichia coli. Biotechnol. Bioeng. 2016, 113, 2670-2675. [CrossRef]

51. Sugimoto, N.; Engelgau, P.; Jones, A.D.; Song, J.; Beaudry, R. Citramalate synthase yields a biosynthetic pathway for isoleucine and straight- and branched-chain ester formation in ripening apple fruit. Proc. Natl. Acad. Sci. USA 2021, 118, e2009988118. [CrossRef] [PubMed]

52. Nishio, K.; Mizushima, T. Structural and biochemical characterization of mitochondrial citrate synthase 4 from Arabidopsis thaliana Acta Crystallogr. Sect. F Struct. Biol. Commun. 2020, 76, 109-115. [CrossRef] [PubMed]

53. Le, A.; Lane, A.N.; Hamaker, M.; Bose, S.; Gouw, A.; Barbi, J.; Tsukamoto, T.; Rojas, C.J.; Slusher, B.S.; Zhang, H.; et al. GlucoseIndependent glutamine metabolism via TCA cycling for proliferation and survival in B cells. Cell Metab. 2012, 15, 110-121. [CrossRef]

54. Chen, Y.; Fu, Z.; Zhang, H.; Tian, R.; Yang, H.; Sun, C.; Wang, L.; Zhang, W.; Guo, Z.; Zhang, X.; et al. Cytosolic malate dehydrogenase 4 modulates cellular energetics and storage reserve accumulation in maize endosperm. Plant Biotechnol. J. 2020, 18, 2420-2435. [CrossRef] [PubMed] 